\title{
International Valuation Standards (IVS) - Bedarf, Reichweite, Würdigung, diskutiert anhand der Bewertung von Unternehmen und Goodwill
}

\author{
Wolfgang Ballwieser
}

Eingegangen: 23. Dezember 2019 / Angenommen: 21. Februar 2020 / Online publiziert: 2. März 2020

(C) Der/die Autor(en) 2020

Zusammenfassung Der Beitrag zielt auf eine Bedarfsanalyse des International Valuation Standards Council (IVSC) und der von ihm entwickelten International Valuation Standards (IVS) aus deutscher Sicht. Die akademische Beschäftigung mit IVSC und IVS ist bisher rudimentär und erschöpft sich weitgehend in einer Wiedergabe von Standards. Diese Sichtweise zu erweitern ist das übergeordnete Ziel des Beitrags. Betrieben wird die Analyse für die Unternehmens- und Goodwillbewertung, denn zur Unternehmensbewertung existieren IVS und zur Goodwillbehandlung äußert sich das IVSC. Dies erlaubt einen Vergleich mit der ausdifferenzierten Unternehmensbewertung in Deutschland und mit den ökonomischen Resultaten zum Informationswert der Wertminderungen von Goodwill.

Schlüsselwörter International Valuation Standards · Unternehmensbewertung · Goodwill · Impairment-only Approach

JEL-Classifikation M40 - M41 · M48

W. Ballwieser $(\bowtie)$

Fakultät für Betriebswirtschaft, Ludwig-Maximilians-Universität München, Ludwigstr. 28

RG, 80539 München, Deutschland

E-Mail: ballwieser@bwl.lmu.de 


\title{
International Valuation Standards (IVS)—Demand, Range, Critical Appraisal, Discussed in Regard to Business and Goodwill Valuation
}

\begin{abstract}
This study aims at a demand analysis of the International Valuation Standards Council (IVSC) and its International Valuation Standards (IVS) from a German point of view. The academic analysis of IVSC and IVS is rudimentarily and narrows down widely to repetition of standards. This perception to broaden is the overriding aim of the paper. Pursued is the analysis of business and goodwill valuation, since IVS for business valuation and IVSC papers for impairment tests of goodwill exist. This allows to compare with the sophisticated business valuation in Germany and with empirical results about the information value of goodwill impairments.
\end{abstract}

Keywords International Valuation Standards · Business valuation · Goodwill · Impairment-only approach

\section{Problemstellung und Gang der Untersuchung}

In den letzten Jahrzehnten mehrten sich Aktivitäten bei der Regulierung von Unternehmen, die von nichtstaatlichen Organisationen initiiert wurden und zu Beginn nicht auf nationaler oder EU-Gesetzgebung beruhten. Als prominentes Beispiel solcher Organisationen sei das 1973 gegründete International Accounting Standards Committee (IASC) mit Sitz in London genannt. Die Initiative ging von den Instituten der Accountants in Großbritannien aus (Kleekämper 2000, S. 470). Ziel des IASC war die Entwicklung international anerkannter, harmonisierter und damit vergleichbarer Rechnungslegungsstandards. Im Jahr 2000 wurde es bei einer Neuorganisation in International Accounting Standards Board (IASB) umbenannt, soll die früheren Standards anpassen und neue International Financial Reporting Standards (IFRS) entwickeln. Gegenwärtig werden die IFRS in 166 Rechtssystemen verwendet (IFRS 2019). Lange Zeit hat Deutschland nur begrenztes Interesse an den Standards erkennen lassen, was sich spätestens mit Übernahme der IFRS in EU-Recht im Jahr 2002 änderte.

Als zweites Beispiel sei die 1983 als Nachfolger einer gleichnamigen US-amerikanischen Organisation von 1973 gegründete International Organization of Securities Commissions (IOSCO) mit Sitz in Madrid erwähnt. Ihr Ziel ist die Förderung weltweit einheitlicher Standards für die Börsen- und Wertpapierzulassung. Sie empfahl im Jahr 2000 ihren Mitgliedern, die IAS bzw. IFRS für die nationale Börsennotierung zuzulassen. Seit 2005 muss insbesondere jede kapitalmarktorientierte Muttergesellschaft mit Sitz in der EU einen Konzernabschluss nach IFRS erstellen. Die Mitglieder der IOSCO regulieren derzeit mehr als 95\% der weltweiten Wertpapiermärkte in über 115 Rechtssystemen und umschließen alle größeren Schwellenländer (IOSCO 2019).

In diese Reihe fügt sich das 1981 in Melbourne gegründete, in London ansässige, seit 2003 in Illinois in den USA eingetragene International Valuation Standards Committee (IVSC) ein, das 2008 in International Valuation Standards Council (er- 
neut IVSC) umbenannt wurde. Das Gremium ist als nichtstaatliche Organisation UN-Mitglied und arbeitet mit Organisationen wie dem IASB zusammen, um weltweit einheitliche Bewertungsstandards zu generieren und deren einheitliche Anwendung zu fördern. Im November 2019 berichtete dessen Geschäftsführer auf einer Konferenz in Mailand von 138 Mitgliedsorganisationen, die mehr als 340.000 Berufsorganisationen umschließen, und die Anwendung von International Valuation Standards (IVS) in über 103 Ländern (Talbot 2019).

Ökonomisch zu begründen versuchen lassen sich diese Aktivitäten mit der internationalen Tätigkeit vieler Unternehmen, dem Ziel nach verstärkter Transparenz, u. a. aufgrund der Vergleichbarkeit von Abschlüssen, speziell von börsennotierten Unternehmen oder von Mitgliedern solcher Branchen, deren Vertrauenswürdigkeit systemrelevant erscheint, und damit verbundenen geringeren Transaktions- und Risikokosten für Kapitalgeber. Dies zu messen ist keine leichte Aufgabe, kann aber hier unbehandelt bleiben. Im vorliegenden Beitrag stellen wir vielmehr erstens die Frage, ob das IVSC eine den vorher genannten Institutionen vergleichbare Entwicklung nehmen und wirtschaftliche Bedeutung erhalten könnte.

Die Fragestellung ist ökonomisch und politisch interessant und erfährt bisher keine Behandlung in der Literatur. Letztere erschöpft sich in meist kursorischen Hinweisen auf die Existenz des IVSC und - tiefergehend - in früheren Vergleichen von IVS mit IFRS und Regeln zur Immobilienbewertung. Nach unserem Wissen gibt es bisher auch keine Auseinandersetzung mit den aktuellen IVS 2020, was angesichts der in jüngster Zeit erkennbaren Entwicklungsdynamik unbefriedigend ist. Da dieses Regelwerk sich jedoch auf sehr unterschiedliche Vermögenswerte bezieht und diese schon aus Platzgründen nicht gleichermaßen sorgfältig zu diskutieren sind, beschränken wir uns im Folgenden auf die Bewertung von Unternehmen und die Folgebewertung von aktiviertem Goodwill. Hierzu sind zwei Gründe maßgebend: Zum einen existiert in Deutschland ein breites Fundament an ausdifferenzierter Theorie, Praxis und Rechtsprechung zur Unternehmensbewertung. Damit lassen sich die IVS zur Unternehmensbewertung an diesem Wissen spiegeln und würdigen. Deshalb fragen wir zweitens, welche Schlussfolgerungen sich hinsichtlich der potentiellen Verbindlichkeit von IVS oder ihres freiwilligen Orientierungsnutzens für Deutschland ziehen lassen, sprich: welcher Bedarf an IVS besteht.

Zum anderen basiert die Folgebewertung von Goodwill auf Unternehmensbewertungskalkülen und schafft bereits insofern eine Verbindung zum erstgenannten Bereich. Zugleich ist sie in ihrem Informationsnutzen umstritten und führte in den USA 2019 zu einer Invitation to Comment (FASB 2019a). Das IASB verfolgt diese Argumentation und will im 2. Quartal dieses Jahres ein Diskussionspapier vorlegen. Es will (mit knappem Mehrheitsentscheid im letzten Jahr) die planmäßige Abschreibung nicht zur Diskussion stellen, sondern den Impairment-only Approach (IOA) beibehalten und verbessern. In diese Diskussion bringt sich interessanterweise auch das IVSC mit drei Perspective Papers ein, von denen bisher zwei, das letzte am 23. Dezember 2019, erschienen sind (IVSC 2019g, 2019h). Deshalb fragen wir drittens, wie ökonomisch begründet die aktuellen, die Rechnungslegungspolitik des IASB vermutlich (mit-)beeinflussenden Argumente des IVSC in diesen Unterlagen sind.

Keine der drei Fragen ist bisher in der Literatur angemessen behandelt. Zwar gibt es zwei der Themenstellung etwas verwandte Quellen (Gonnella und Talarico 2018; 
Tönnes 2019). Sie behandeln aber nur Teilaspekte unserer Fragen und bauen auf IVS 2017 statt IVS 2020 auf. Der Beitrag verspricht insofern Neuigkeitswert.

Abschn. 2 gibt einen kurzen Überblick über die Struktur des IVSC und einen längeren über IVS 2020 hinsichtlich Struktur, Inhalt und Länge, um eine Basis für die Diskussion zur Unternehmens- und Goodwillbewertung zu schaffen. Abschn. 3 skizziert das Entwicklungskonzept von IVS, ihre Bindungswirkung und deren maßgebliche Gestalter, bevor die Abschn. 4 und 5 die Unternehmens- und die Thesen zur Goodwillbewertung behandeln und anhand explizierter Kriterien würdigen. Abschn. 6 bündelt die Ergebnisse.

\section{IVSC und IVS 2020 im Überblick}

\subsection{IVSC}

Politisch zeigt sich die Entwicklung des IVSC aus dem angelsächsischen Bereich, mit starker Kapitalgeber-, speziell Investorensicht, getrieben. Börsennotierte Unternehmen sind zentral, wenn auch nicht allein im Blick. Das IVSC widmet sich der Bewertung von Vermögenswerten, Unternehmen und Finanzinstrumenten. Es stützt sich organisatorisch auf drei Technical Boards (im Folgenden behalten wir die englischen Ausdrücke bei, um unscharfe Übersetzungen zu vermeiden): Tangible Assets Board, Business Valuation Board und Financial Instruments Board, die ein Standards Review Board überwacht, um widerspruchsfreie und vollständige Regelungen zu ermöglichen. Ein Advisory Forum repräsentiert Mitgliedsorganisationen und regt zu behandelnde Themen an. Ein Membership and Standards Recognition Board widmet sich der Verbreitung der IVS und ein Board of Trustees soll die unabhängige Aufsicht über das IVSC ermöglichen. 2019 wurde ein Europe Membership and Standards Recognition Board in Angriff genommen, das seit Ende Februar 2020 auf der Website zu finden ist.

Die Geschichte des IVSC zeigt personelle und inhaltliche Verflechtungen mit dem IASB. So wurde im Jahr 2012 Sir David Tweedy nach über zehnjähriger Tätigkeit als IASB-Vorsitzender Mitglied des IVSC und Vorsitzender des Board of Trustees bis Oktober 2019. Mit Alistair Darling, einem früheren britischen Finanzminister, übernahm ein weiterer Schotte sein Amt. Und im März 2014 veröffentlichten die IFRS Foundation und das IVSC ein gemeinsames Protokoll, mit dem die Kooperation von IASB and IVSC zum Zwecke einer konsistenten Bewertung von Fair Values angestrebt wurde (IVSC 2014). Auch im Bereich der Immobilienbewertung suchte man schon zuvor den Kontakt zwischen den Gremien.

\subsection{IVS 2020}

\subsubsection{Struktur, Themen und Länge der Standards 2020}

Die letzte gedruckte Ausgabe der IVS stammt von 2017 (IVSC 2017), für die im Juli 2018 (IVSC 2018) und im Januar und März 2019 (IVSC 2019a, 2019b) Änderungsvorschläge zur Kommentierung veröffentlicht wurden. Die Kommentierungen 
sind nur zum erst- und letztgenannten Exposure Draft auf der Website des IVSC einsehbar (IVSC 2019c, 2019d). Hingegen gibt es eine Basis for Conclusions (IVSC 2019e), die auf alle drei Änderungsvorschläge eingeht. Allein online steht seit August 2019 die aktuelle Version der IVS, mittlerweile ohne Jahreszahl im Titel, aber mit Anwendungsbereich ab 31. Januar 2020, zur Verfügung (https://www.ivsc.org/ news/article/ivs-updated). Wir nennen sie hier deshalb IVS 2020. Deutlich wird die Geschwindigkeit, mit der das IVSC in jüngster Zeit voranschreitet.

Die IVS 2020 enthalten auf 132 Seiten neben Introduction, Glossary und Framework fünf General Standards, sieben Asset Standards und einen Index. Die General Standards beschäftigen sich mit Scope of Work (IVS 101), Investigations and Compliance (IVS 102), Reporting (IVS 103), Bases of Value (IVS 104) und Valuation Approaches and Methods (IVS 105). Die Asset Standards behandeln Business and Business Interests (IVS 200), Intangible Assets (IVS 210), Non-Financial Liabilities (IVS 220), der gegenüber IVS 2017 neu ist, Plant and Equipment (IVS 300), Real

Tab. 1 Seitenlänge der IVS 2020

\begin{tabular}{|c|c|c|c|}
\hline Thema & Seitenzahl & $\begin{array}{l}\text { Prozentualer Seitenanteil } \\
\text { (mit Rundungsfehlern) } \\
(\%)\end{array}$ & $\begin{array}{l}\text { Wie links, ohne Leerseiten } \\
\text { Introduction und Index } \\
(\%)\end{array}$ \\
\hline $\begin{array}{l}\text { Valuation Approaches } \\
\text { and Methods (IVS 105) }\end{array}$ & 21 & 16 & 18 \\
\hline $\begin{array}{l}\text { Intangible Assets (IVS } \\
210 \text { ) }\end{array}$ & 17 & 13 & 14 \\
\hline $\begin{array}{l}\text { Bases of Value (IVS } \\
104)\end{array}$ & 13 & 10 & 11 \\
\hline $\begin{array}{l}\text { Business and Business } \\
\text { Interests (IVS 200) }\end{array}$ & 12 & 9 & 10 \\
\hline $\begin{array}{l}\text { Development Property } \\
\text { (IVS 410) }\end{array}$ & 11 & 8 & 9 \\
\hline $\begin{array}{l}\text { Non-Financial Liabili- } \\
\text { ties (IVS 220) }\end{array}$ & 10 & 8 & 8 \\
\hline $\begin{array}{l}\text { Financial Instruments } \\
\text { (IVS 500) }\end{array}$ & 9 & 7 & 8 \\
\hline Index & 9 & 7 & - \\
\hline $\begin{array}{l}\text { Real Property Interests } \\
\text { (IVS 400) }\end{array}$ & 7 & 5 & 6 \\
\hline $\begin{array}{l}\text { Plant and Equipment } \\
\text { (IVS 300) }\end{array}$ & 7 & 5 & 6 \\
\hline $\begin{array}{l}\text { Scope of Work (IVS } \\
101)\end{array}$ & 3 & 2 & 3 \\
\hline Glossary & 3 & 2 & 3 \\
\hline Reporting (IVS 103) & 2 & 2 & 2 \\
\hline $\begin{array}{l}\text { Investigations and Com- } \\
\text { pliance (IVS 102) }\end{array}$ & 2 & 2 & 2 \\
\hline Framework & 2 & 2 & 2 \\
\hline Introduction & 2 & 2 & - \\
\hline Leer & 2 & 2 & - \\
\hline Summe & 132 & 100 & 100 \\
\hline
\end{tabular}


Property Interests (IVS 400), Development Property (IVS 410) und Financial Instruments (IVS 500) (zum Überblick nach IVS 2017; s. Tönnies 2019, S. 430-443; Gonnella und Talarico 2018, S. 105-110).

Sortiert man die Standards nach ihrer Seitenlänge, so ergeben sich die Daten aus Tab. 1.

Die Seitenlänge sagt zwar nichts über den materiellen Inhalt. Aber es zeigt sich, dass die Themen von Unternehmens- und Goodwillbewertung in den drei längsten Standards mit rd. 40\% Seitenanteil angesiedelt sind, nämlich in IVS 104, 105 und 210 .

\subsubsection{Wesentliche Inhalte von IVS 104, 105 und 210 im Überblick}

In IVS 104.20.1(a) werden in einer ersten Liste sechs IVS-Bases of Value angegeben: Market Value, Market Rent, Equitable Value, Investment Value/Worth, Synergistic Value und Liquidation Value. IVS 104.20.1(b) nennt in einer beispielhaften Liste noch verschiedene Fair-Value-Konzepte als ,other bases of value“. Sie stammen von IASB, OECD, US-Internal Revenue Service, US-Model Business Corporation Act und Canadian case law. Vergleicht man die Wertgrundlagen mit Deutschland, gilt Folgendes:

Synergistic Value und Liquidation Value entsprechen dem theoretischen Verständnis in Deutschland.

Market Value definiert IVS 104.30.1 als ,the estimated amount for which an asset or liability should exchange on the valuation date between a willing buyer and a willing seller in an arm's length transaction, after proper marketing and where the parties had each acted knowledgeably, prudently and without compulsion." Das klingt nach gemeinem Wert im Sinne des Steuerrechts.

Equitable Value ist gemäß IVS 104.50.1, ,the estimated price for the transfer of an asset or liability between identified knowledgeable and willing parties that reflects the respective interests of those parties." Man könnte ihn als gerechten Preis bezeichnen. Wolf-Achim Tönnes sieht Anklänge zum aus der Unternehmensbewertung bekannten Schiedswert (Tönnes 2019, S. 445).

Die beiden zuletzt genannten Konzepte unterscheiden sich wie folgt: Der Equitable Value ist auf zwei identifizierte und damit konkrete Parteien bezogen, während sich der Market Value auf Marktteilnehmer allgemein (,generally“) bezieht (IVS 104.50.2).

IVS 104.60.1 definiert: „Investment Value is the value of an asset to a particular owner or prospective owner for individual investment or operational objectives." Das entspricht dem Grenzpreis als potentiellem Preis (Moxter 1983, S. 5) bzw. dem Entscheidungswert (Coenenberg und Sieben 1976, Sp. 4065).

Die erste Liste überzeugt aus mehreren Gründen nicht: (1) die Angabe einer Rente als Wert vernachlässigt den Unterschied von Bestands- und Flussgrößen (man könnte im Übrigen auch Umsatz oder Rohgewinn zur Wertbasis erheben), (2) die eigenständige Nennung von Synergistic Value unterschlägt, dass er Bestandteil von Investment Value sein kann (und oft sein wird), und lässt offen, wozu er separiert werden muss, und (3) Wert und Preis werden bei Market Value und Equitable 
Value vermengt. Schließlich ist die beispielhafte zweite Liste einseitig, weil sie überwiegend an USA und IASB orientiert ist.

IVS 105 beschreibt nach Einführung Markt- (S. 30-36), Discounted Cash Flow (DCF)- (S. 36-44) und Kostenbewertungen (S. 44-47), bevor Abschreibung und Veralterung behandelt werden (S. 47-49). Neu gegenüber IVS 2017 ist eine halbe Seite zu einem Valuation Model (S. 49).

Gemäß IVS 105.10.8 werden Preisinformationen von einem aktiven Markt als stärkster Beweis für den Wert angesehen, auch wenn kein Wertansatz unter allen Umständen anwendbar ist. In einzelnen Fällen könnten subjektive Anpassungen von Preisinformationen sogar verboten sein (,Some bases of value may prohibit ...“). Hier hätten Bewerter ,a responsibility to consider“ (Glossary.20.6).

IVS 210 beschäftigt sich erneut mit Markt-, DCF- und Kostenbewertungen, bevor Special Considerations for Intangible Assets (S. 75), Discount Rates/Rates of Return for Intangible Assets (S. 75 f.), Intangible Asset Economic Lives (S. 76-78) und Tax Amortisation Benefit (S. 78f.) behandelt werden.

Goodwill wird als Synonym von Going concern value bereits in IVS 200.20.1 erwähnt und findet sich in mehreren Absätzen von IVS 210 (S. 63-65, 68f.) und IVS 300 (S. 94). Der Index ist diesbezüglich unvollständig. Zwar gibt es Hinweise auf ein Impairment, aber nicht auf einen Impairment-only Approach (IOA).

\section{Entwicklungskonzept von IVS, deren Bindungswirkung und maßgebliche Gestalter}

Die Einführung in IVS 2020 nennt Zielsetzung des IVSC und Eigenschaften von IVS: „Our primary objective is to build confidence and public trust in valuation by producing standards and securing their universal adoption and implementation for the valuation of assets across the world. (...) The International Valuation Standards (IVS) are standards for undertaking valuation assignments using generally recognised concepts and principles that promote transparency and consistency in valuation practice. The IVSC also promotes leading practice approaches for the conduct and competency of professional valuers." (IVS 2020, S. 1).

Es geht mithin beim Entwicklungskonzept von IVS um ,generally recognised concepts and principles“ und „leading practice approaches“. Dazu passen ein „,due process“ und die ,consultation with stakeholders“" sowie die Verbindung mit Institutionen, die standardsetzende Funktion in den Finanzmärkten haben (IVS 2020, S. 1).

Erkennbar wird ein Bottom-up-Ansatz mit der Suche nach „,best practice“. Geregelt werden sollen solche Themen, die Aufmerksamkeit erheischen (,require consideration“; IVS 2020, S. 1). Das erinnert sehr an das Vorgehen des IASC nach seiner Gründung im Jahr 1973.

Die Bindungswirkung für IVS-Anwender wird wie folgt umschrieben: ,The IVS consist of mandatory requirements that must be followed in order to state that a valuation was performed in compliance with the IVS." (IVS 2020, S. 2; Hervorhebung Verf.). 
Nach IVS Framework.60.2 kann es zwingende Abweichungen von IVS geben, die Vorrang haben: „The requirement to depart from IVS pursuant to legislative, regulatory or other authoritative requirements takes precedence over all other IVS requirements.“ Das führt zu einer Modifikation des vorigen Statements: „A valuer may still state that the valuation was performed in accordance with IVS when there are departures in these circumstances." (IVS Framework.60.1; Hervorhebung Verf.). Das ist bei der Unternehmensbewertung in ähnlicher Weise bekannt, wenn die dem Berufsrecht unterworfenen Wirtschaftsprüfer ein Bewertungsgutachten statt nach dem für sie grundsätzlich verbindlichen IDW S 1 als in Anlehnung an IDW S 1 erstellt ausgeben. Die Güte von IDW S 1 kann hierbei offen bleiben.

Aus dem Bottom-up-Ansatz resultieren Mitgliederstruktur und Finanzierung des IVSC. Der Jahresbericht 2018-19 lässt 139 Mitgliedorganisationen erkennen. Darunter finden sich die WP-Gesellschaften Deloitte, EY, KPMG, PwC, BDO und Grant Thornton, Verbände wie AICPA und ICAEW, der Finanzdienstleister Bloomberg, die Bewertungsgesellschaft Duff \& Phelps, das Finanzinstitut Société Générale, die Alliance Manchester Business School der University of Manchester, die Levanthal School of Accounting der University of Southern California, das Mongolian Institute of Certified Appraisers und das Ministry of Land Reform Namibia (IVSC 2019f, S. 28). Das lässt sehr unterschiedliche Interessenten und Bewertungskompetenz erkennen. 102 Mitglieder haben einen Immobilienschwerpunkt, 75 fokussieren auf Unternehmensbewertung und 29 auf Finanzinstrumente (IVSC 2019f, S. 26). Deutschland stellt mit der European Association of Certified Valuators and Analysts (EACVA) und der auf Immobiliengutachten spezialisierten HypZert GmbH nur zwei Mitglieder, und dies erst ab 2018. Die Bilanzsumme zum 31. März 2019 beträgt rd. 1 Mio. $£$ bei einem Eigenkapital von rd. 75\%. Ein Vergleich mit dem IASB zeigt erhebliche Größenunterschiede. Dessen Bilanzsumme beläuft sich zum 31. Dezember 2018 auf rd. 40 Mio. $£$ bei einem Eigenkapital von rd. $85 \%$.

Das 14-köpfige Board of Trustees weist neun Mitglieder aus USA, Großbritannien und Kanada auf. Das 13-köpfige Standard Review Board hat mit neun Mitgliedern aus USA, Großbritannien und Australien eine ähnliche Struktur. Beide Boards sind vorrangig angelsächsisch geprägt. Ihre Mitglieder haben oft herausragende Posten in WP-Gesellschaften und -Verbänden oder in Regulierungsinstitutionen inne gehabt.

\section{Unternehmensbewertung in IVS 2020}

\subsection{Wesentliche Aussagen zur Unternehmensbewertung}

Wie in Abschn. 2.2.2 erwähnt beschreibt IVS 105 Markt-, DCF- und Kostenbewertungen. Für die Unternehmensbewertung interessieren nur die beiden ersten Ansätze. Hierzu liest man zum ersten Ansatz in IVS 105.20.1: „The market approach provides an indication of value by comparing the asset with identical or comparable (that is similar) assets for which price information is available." Anschließend werden die Comparable Transactions Method, die Guideline Publicly-traded Comparable Method und Other Market Approach Considerations ,that may form part of a market approach valuation“ vorgestellt. Zu letzteren werden Discount for Loss of Market- 
ability (DLOM), Control Premiums, Discount for Loss of Control (DLOC) und Blockage Discounts gezählt.

Der schon erwähnte Bottom-up-Ansatz zeigt sich deutlich in der Feststellung: „Anecdotal or ,rule-of-thumb valuation benchmarks are sometimes considered to be a market approach. However, value indications derived from the use of such rules should not be given substantial weight unless it can be shown that buyers and sellers place significant reliance on them." (IVS 105.30.16).

Zum zweiten Ansatz findet man in IVS 105.40.1: „Under the income approach, the value of an asset is determined by reference to the value of income, cash flow or cost savings generated by the asset.“ Es folgen Ausführungen zu DCF-Verfahren mit Detailplanungsphase und Terminal Value inklusive Market Approach/Exit Value und Salvage Value/Disposal Cost.

Zum bei DCF-Verfahren notwendigen Diskontierungszins heißt es in IVS 105.50.31: „Valuers may use any reasonable method for developing an appropriate discount rate. While there are many methods for developing a discount rate or determining the reasonableness of a discount rate, a non-exhaustive list of common methods includes:

(a) a capital asset pricing model (CAPM),

(b) a weighted average cost of capital (WACC),

(c) observed or inferred rates/yields,

(d) a build-up method (generally used only in the absence of market inputs).“

Direkt anschließend folgt in IVS 105.50.32: „Valuers should consider corroborative analyses when assessing the appropriateness of a discount rate. A non-exhaustive list of common analyses should include:

(a) an internal rate of return (IRR),

(b) a weighted average return on assets (WARA),

(c) value indications from other approaches, such as market approch, or comparing implied multiples from the income approach with guideline company market multiples or transaction multiples."

Diese Ausführungen reichen, um einen ersten Eindruck von den Inhalten von IVS $105 \mathrm{zu}$ erlangen.

\subsection{Würdigung}

\subsubsection{Würdigungskriterien}

In Deutschland unterscheidet man nach den Funktionen einer Unternehmensbewertung (Sieben 1966, S. 8; 1977, S. 28-30). Es gilt das Zweckadäquanzprinzip (Moxter 1983, S. 5). Das Institut der Wirtschaftsprüfer (IDW) hat sich dem angeschlossen (IDW 2008), auch wenn die inhaltlichen Aussagen in theoretischer und IDW-Literatur nicht deckungsgleich sind. Insbesondere ist der theorieorientierten Literatur der objektivierte Unternehmenswert des IDW fremd (Ballwieser und Hachmeister 2016, S. 3-5). Unabhängig davon lassen sich die Ermittlungen von Grenzpreisen (oder Entscheidungswerten), von Vermittlungswerten, von Argumentationswerten sowie von 
handels-, steuer- und gesellschaftsrechtlich normierten Werten unterscheiden. Ein erstes Würdigungskriterium ist deshalb, welche Funktionen oder Bewertungszwecke in IVS 105 angesprochen werden. Ein zweites Kriterium ist die Ausführlichkeit und Anwendungsunterstützung sowie der Allgemeinheits- bzw. Konkretisierungsgrad der Ausführungen. Schließlich interessieren deren Richtigkeit und Widerspruchsfreiheit.

\subsubsection{Resultate}

Zwecke oder Funktionen der Unternehmensbewertung werden nicht weiter erläutert. Die Einführung besagt lediglich, dass die General Standards für alle Arten von Vermögenswerten und jeden Bewertungszweck entwickelt werden (IVS 2020, S. 2). Hier fällt bereits auf, dass Schulden ausgenommen sind, obwohl auch sie zu bewerten sind. Das Glossar verweist darauf, dass gewöhnliche Zwecke die Finanzberichterstattung, die steuerliche Rechnungslegung und die Unterstützung von Prozessen, Transaktionen und gesicherten Kreditvergaben einschließen, ohne darauf beschränkt zu sein (IVS 2020, S. 4). Nach IVS 101.20.1 muss jede Bewertung zweckgerecht erfolgen. Der Zweck bestimmt die Bewertungsbasis (IVS 101.20.3(f) und (g)). Über alle Standards hinweg ist der Verweis auf den Bewertungszweck gegenwärtig. Jedoch unterbleibt jegliche Systematisierung und daraus folgender Hinweis auf Bewertungsverfahren. Stattdessen finden sich beispielhafte Aufzählungen von Anlässen, die mit ganz verschiedenen Bewertungszwecken verbunden sein können. So erwähnt IVS 200.20.4 Unternehmenskäufe und -verkäufe, Fusionen, Besteuerung, Prozesse, Insolvenzverfahren und Rechnungslegung. Dass bei Käufen Grenzpreise, Schiedswerte oder Argumentationswerte gefragt sein können, in welcher Beziehung sie zueinander stehen und welche Bewertungsverfahren geeignet sein können, wird nicht behandelt.

Rechtfertigen könnte man das vielleicht mit dem in der Einführung zu findenden Hinweis, dass bestimmte Ansichten der Standards ,do not direct or mandate any particular course of action, but provide fundamental principles and concepts that must be considered in undertaking a valuation" (IVS 2020, S. 2). Auch verlangen General Standards, zu denen die für die Unternehmensbewertung maßgeblichen IVS 104 und 105 zählen, Beschränkungen auf das Gemeinsame aller Bewertungen, mit denen sich die IVS beschäftigen.

Dies vorausgeschickt, fallen doch einige Sätze auf, die Optionen beschreiben (,es mag ...") und Existenzen feststellen (,es gibt ...“). Beispielhaft verwiesen sei auf DLOMs. Hier heißt es: „DLOMs may be quantified using any reasonable method, but are typically calculated using option pricing models, studies that compare the value of publicly-traded shares and restricted shares in the same company, or studies that compare the value of shares in a company before and after an initial public offering.“ (IVS 105.30.17(a)). Ähnlich heißt es an anderer Stelle: „Control Premiums and DLOCs may be quantified using any reasonable method, but are typically calculated based on ..." (IVS 105.30.17(b)). Schließlich liest man bei blockage discounts: „Blockage discounts are sometimes applied when ...“(IVS 105.30.17(c)). Entsprechende Aufzählungen finden sich für Cash Flows (IVS 105.50.16) und s. oben die Zitate im letzten Abschnitt - für Zinssätze (IVS 105.50.31f.). 
Dieser Ansatz ist für den Aussagegehalt einer Bestätigung, dass - jenseits von regulatorisch vorrangigen Anforderungen - sämtliche IVS eingehalten worden sind, sehr kritisch, denn was fängt ein Adressat dieser Botschaft damit an? Schließlich behauptet doch IVS 2020, S. 2: „The IVS consist of mandatory requirements that must be followed (...)“. „Mandatory requirements“ lassen strikte Verpflichtungen oder aber die Nennung der zu erfüllenden Bedingungen für die Auswahl einer bestimmten Option aus einer Alternativenmenge erwarten. Beides fehlt. Tönnes sieht hierbei auch zu Recht die Verfehlung des Ziels einer internationalen Vergleichbarkeit der Standards (Tönnes 2019, S. 449). Zugleich wird an anderer Stelle konstatiert, dass die IVS sich stärker prozeduralen und formalen anstelle von inhaltlichen Aspekten widmen. Sie repräsentierten nur eine Menge an Minimalanforderungen, die Bewerter zu beachten haben, so dass die subjektive Natur der Unternehmensbewertung von besonderer Bedeutung sei (Gonnella und Talarico 2018, S. 102). Es handele sich um eine prinzipien-orientierte Selbstregulierung ohne Sanktionsmöglichkeiten. Dem Bewerter fehle die direkte Handreichung (Gonnella und Talarico 2018, S. 113).

Aus strategischer Sicht des IVSC ist diese Vorgehensweise verständlich, da die Vermeidung rigider Verpflichtungen die Aufnahme vieler Mitglieder, die in ihrem jeweiligen Land unterschiedlichsten Bedingungen ausgesetzt sind und unterschiedlichste Aufgaben erfüllen, in das IVSC erlaubt. Die schon erwähnte Bewertungsgesellschaft Duff \& Phelps, das Mongolian Institute of Certified Appraisers und das Ministry of Land Reform Namibia dürften insoweit kaum vergleichbar sein. Auch passt dieses Vorgehen zum Bottom-up-Ansatz und der Suche nach „best practice“, freilich um den Preis fehlender Theorieunterlegung, fehlender eventueller Aufdeckung von Widersprüchen bei gleichzeitiger Stützung auf verschiedene Systeme und damit verbundenen Kontexten und fehlender Handlungsunterstützung bei speziellen Wahlmöglichkeiten des Bewerters. Schon das Fehlen einer aussagekräftigen Systematisierung von Bewertungszwecken behindert eine zweckgerechte Ableitung von Handlungsempfehlungen für die Bewertung, wie sie insbesondere in Deutschland erkennbar ist. Zugleich wird der potentielle Wert einer Bestätigung, die IVS eingehalten zu haben, beeinträchtigt.

Zur Richtigkeit und Widerspruchsfreiheit mögen zwei Beispiele genügen, auch wenn sie nicht repräsentativ für den Gesamttext sind. Falsch ist die Aussage in IVS 105.50.23: „The constant growth model assumes that the asset grows (or declines) at a constant rate into perpetuity. "Es wachsen in diesem Modell die zu diskontierenden Cash Flows (eine Flussgröße), die als erwartete Zahlungen an die Kapitalgeber zu verstehen sind, nicht die Vermögenswerte (Bestandsgrößen). Nicht gleichgewichtig sind die Gegenüberstellungen von CAPM und WACC in IVS 105.50.31, weil das erste Modell Bestandteil des WACC sein kann (und oft sein dürfte).

Für die ersten zwei Fragestellungen dieses Beitrags ergeben sich danach folgende Schlussfolgerungen: Bei Extrapolation des jetzigen Zustands der IVS 2020 kann dem IVSC eine dem IASC oder der IOSCO vergleichbare Entwicklung und Bedeutung nicht zukommen. Es fehlen hierzu, wie bei den IFRS, nicht nur die Übernahme in nationales oder EU-Recht, die Manpower und die Finanzmittel, sondern auch der Versuch der Ausrichtung der Bewertungsstandards auf ein inhaltliches Ziel, wie es den IFRS vergleichbar ist. Das IVSC befindet sich noch im Stadium des Sammelns und Aufzählens relativ allgemeingehaltener Bewertungsgrundlagen und -verfahren 
ohne stringente Ordnung und Prüfung auf Ableitbarkeit, Widerspruchsfreiheit oder Komplementarität. Damit ist auch unklar, wie relativ gesichertes Wissen aus höher entwickelten Ländern oder von bewertungstechnisch kompetenten Mitgliedern des IVSC in schwächer entwickelte Länder weitergegeben werden kann. Angestrebte Harmonisierung und Vergleichbarkeit der Bewertung wird nach jetzigem Stadium nicht erreicht. Die potentielle Verbindlichkeit von IVS oder ihres freiwilligen Orientierungsnutzens schafft deshalb auch für Unternehmensbewerter in Deutschland, mit Ausnahme einer Unterstützung der Argumentationsfunktion, keinen Mehrwert. Aus jetziger Sicht besteht insofern kein Bedarf an IVS.

\section{Die Diskussion zur Goodwillbewertung}

\subsection{Anlass der Diskussion}

\subsubsection{USA}

Der IOA zur Goodwill-Folgebewertung ist seit vielen Jahren ein kontrovers, nunmehr erneut aktuell diskutiertes Thema. Der Streit resultiert aus der Tatsache, dass die Goodwillhöhe in manchen Branchen von vielen Betrachtern als gefährlich empfunden wird (und im Enforcement der Rechnungslegung ein jährlich wiederkehrendes Prüfungsgebiet darstellt), der Wertminderungstest auf zahlreichen, nur schwer objektivierbaren Daten vorgenommen werden muss und Anreize des Managements bestehen können, ihn zu seinen Gunsten ausfallen zu lassen, um Gewinnausweis und Boni nicht zu beeinträchtigen. Auch ist nicht vergessen, dass er 2001 in den USA nicht mit dem Argument der Informationsverbesserung für Kapitalmarktadressaten eingeführt wurde, sondern politischer Preis für die Abschaffung der Methode der Interessenzusammenführung bei der Kapitalkonsolidierung war (Beyer 2015, S. 244; Ramanna 2008). Dem Management eines Unternehmens wurde damit die Möglichkeit der Buchwertfortführung im Konzernabschluss genommen. Diese hatte für Manager den Vorteil, nach einem Unternehmenszusammenschluss oder -erwerb von späteren Abschreibungen auf im Rahmen der Erwerbsmethode aufgelöste stille Reserven von Vermögenswerten verschont zu bleiben. Die Hürde künftiger Gewinnerzielung wurde durch die ab 2001 allein geltende Erwerbsmethode höher. Planmäßige Goodwill-Abschreibungen hätten die Performance des Managements noch weiter beeinträchtigt. Interessanterweise wurde schließlich im Jahr 2014 die planmäßige Abschreibung über 10 Jahre für nicht börsennotierte Unternehmen wieder erlaubt (FASB 2014). Seit 2019 gilt diese Regelung auch für nicht gewinnorientierte Unternehmen (FASB 2018).

Das FASB hat insofern die Diskussion um die Goodwillbehandlung erneut angestoßen, weil die Kritik an ihr seit Jahren nicht verstummt ist. Die Meinung, man müsse sie wenigstens verbessern, u. U. sogar zur planmäßigen Abschreibung zurückkehren, wurde immer lauter artikuliert. Insbesondere wurde der Impairment-Test als sehr kostenträchtig und manipulationsanfällig empfunden, was die Frage nach angemessenem Kosten-Nutzen-Verhältnis und Vertrauenswürdigkeit der Rechnungslegung emporrief. Daraufhin wurde im Juli 2019 eine Einladung zur Kommentierung 
der Goodwill-Bilanzierung vorgelegt (FASB 2019a), das 103 Kommentierungen hervorrief (FASB 2019b).

Blickt man einmal auf die Kommentare der Big 4-WP- und Beratungs-Gesellschaften, die intensiv in den IOA involviert sind, so ist eine sehr unterschiedliche Sicht zu erkennen. Je eine Gesellschaft schlägt eine Verbesserung sowie eine Abschaffung des IOA vor, zwei Gesellschaften lassen die Lösung offen. So spricht sich KPMG gegen die alleinige planmäßige Abschreibung aus und schlägt eine Verbesserung des IOA vor, wenn sich ein Abschreibungsmodell nicht genügend vereinfachen lässt. Die Gesellschaft empfiehlt, unter diesen Umständen den Wertminderungstest von der Berichtseinheit auf das operative Segment zu verlagern. Sollte sich das FASB für die planmäßige Abschreibung entscheiden, sei die gewogene durchschnittliche Nutzungsdauer der erworbenen identifizierbaren Vermögenswerte als Abschreibungsdauer wünschenswert. Der Ansatz stünde auf einem objektiven Maß auf Basis von Informationen, die das Unternehmen sowieso erhebe (FASB 2019b, Comment Letter No. 15, S. 1 f.). EY unterstützt hingegen eine planmäßige Abschreibung und einen Wertminderungstest allein auf Grund eines auslösenden Ereignisses (FASB 2019b, Comment Letter No. 65, S. 1). PwC nennt der Gesellschaft bekannte und ihr gegenüber geäußerte Vor- und Nachteile des IOA und schlägt dem FASB vor, dass es den Input von Nutzern und Aufstellern gewichten möge, wenn es mit seinem Projekt fortfährt (FASB 2019b, Comment Letter No. 18, S. A2). Und Deloitte stellt fest, dass es konzeptionelle Gründe gäbe, Goodwill als abnutzbar und nicht abnutzbar anzusehen, wie es in der Basis for Conclusions von SFAS 142 diskutiert worden sei. Das Board solle eine mit dieser konzeptionellen Basis, die sich nicht verändert habe, zu vereinbarende Entscheidung treffen (FASB 2019b, Comment Letter No. 57, S. $1 \mathrm{f}$.).

Hingegen sprechen sich z. B. die mit 41 und 23 Seiten umfassenden Stellungnahmen des CFA Institute (FASB 2019b, Comment Letter No. 103) und von Duff \& Phelps (FASB 2019b, Comment Letter Letter No. 67, S. 2) für eine Verbesserung des IOA aus, während zwei Professoren der University of Baltimore in einer zweiseitigen Stellungnahme die planmäßige Abschreibung favorisieren (FASB 2019b, Comment Letter No. 20).

\subsubsection{IASB}

Das IASB übernahm den IOA grundsätzlich mit IFRS 3.4 im Jahr 2004 und kennt bis heute davon keine Ausnahme. Es hat im Anschluss an den Post-implementation Review von IFRS 3 ein „Goodwill and impairment project“ gestartet. Nach dessen Kernergebnissen gibt es keine starken neuen Argumente für eine planmäßige Abschreibung, ist diese nur eine pragmatische und kostengünstige Lösung, um Bedenken über die Werthaltigkeit des Goodwill zu mindern, stellt jedoch keine nützliche Information für Adressaten der Rechnungslegung dar und beeinträchtigt den Informationsgehalt des IOA (IASB 2016 und 2015). Akzeptiert man einmal diese Gründe, dann ist die in den USA erlaubte planmäßige Abschreibung nur durch Kosten-Nutzen-Abwägungen zu erklären. Schwer fällt dabei jedoch zu verstehen, weshalb der Informationswert für Investoren bei börsennotierten und nicht börsennotierten Unternehmen unterschiedlich sein soll. Auch kann man nicht davon ausgehen, 
dass Unternehmenskäufe und Fusionen bei nicht börsennotierten Unternehmen zu vernachlässigen sind.

Das IASB verfolgt die Diskussion in den USA, weil es grundsätzlich ein Auseinanderfallen der Regelungen dort und in den Ländern, die IFRS nutzen, nicht begrüßt. Auch sind die Auffassungen im Board sehr geteilt. Es plant, im Februar 2020 ein Diskussionspapier hierzu vorzulegen (https://www.ifrs.org/projects/workplan/goodwill-and-impairment/; zugegriffen: 22.12.2019).

\subsubsection{IVSC}

Das IVSC will mit drei Perspective Papers, von denen zwei erschienen sind (IVSC 2019g, 2019h), die Diskussion von IASB und FASB befruchten. Das erste Paper fragt, ob Goodwill ein abnutzbarer Vermögenswert ist, und verneint dies. Begründet wird es damit, dass bei entgegengesetzter Antwort ein unendlich langes Wachstum von Cash Flows in zur Unternehmensbewertung beliebten DCF-Modellen nicht zu erklären sei (IVSC 2019g, S. 8 f.).

Das zweite Paper geht auf den Informationswert einer Wertminderung von Goodwill ein und fragt, ob Wertminderungen als vor- oder nachlaufendes Kapitalmarktsignal verstanden werden müssen. Es stellt fest, dass die Resultate über den Informationswert im Sinne eines vorlaufenden Signals widersprüchlich sind, und nimmt auf fünf akademische Quellen Bezug, wobei die älteste von Hayn und Hughes (2006) relativiert wird, weil sie sich auf eine Stichprobe vor Einführung von SFAS 142 bezieht (IVSC 2019h, S. 4). Gefolgert wird: ,In summary, while in certain instances goodwill impairments are undoubtably a leading indicator, impairments do not appear to consistently serve as a leading indicator of future cash flows and returns" (IVSC 2019h, S. 4). Deshalb wird folgende Vorgehensweise gewählt: „Rather than attempt to analyse historical observations or draw a consensus from the existing academic studies on the topic, the IVSC has instead analysed the accounting framework to better understand why goodwill impairments in certain situations fail to be a leading indicator. In doing so, we identify four primary reasons for why goodwill impairments often lag market sentiment, and utilise a number of examples to articulate the fact patterns which lead to these outcomes." (IVSC 2019h, S. 3). Die vorrangigen Gründe sind:

a) Impairment-Schutz bei Unternehmenserwerb durch intern generierten Puffer aufgrund selbsterzeugter und nicht aktivierter immaterieller Vermögenswerte,

b) Impairment-Schutz nach Unternehmenserwerb durch Abschreibung erworbener und neuer nicht aktivierter immaterieller Vermögenswerte,

c) übermäßig umfassende und nach außen gerichtete Impairment-Auslöser,

d) Verhaltensaspekte - Abneigung gegen Impairment-Entscheidungen.

Anhand von fünf Tabellen mit Zahlenbeispielen werden die ersten zwei Punkte zu belegen, aber auch zu zeigen versucht, dass planmäßige Abschreibung den Puffer gegen Impairment vergrößert. „Leveraging the conclusions outlined above, one can clearly see how a move to amortise goodwill would severely reduce the information value of the goodwill impairment process and exacerbate the shortcomings of the test (shielded impairment and a lagging indicator)“ (IVSC 2019h, S. 11). 
Das dritte, noch diskutierte Paper soll Verbesserungen des Wertminderungstests behandeln.

Angesichts dieses Vorhabens ist es interessant zu untersuchen, wie sehr die auf politische Beeinflussung gerichteten Papers theoretischen Kenntnissen standhalten. Darauf wird im Folgenden eingegangen, ohne methodische Details zu beschreiben.

\subsection{Theoretische Erkenntnisse}

Die Beiträge der Literatur zu Bedeutung von Goodwill und IOA beschäftigen sich insbesondere mit folgenden Fragestellungen (vgl. a. Boennen und Glaum 2014, S. 11; Glaum 2017, S. 26):

a) dem absoluten und relativen Umfang einer Goodwillaktivierung,

b) den Einflussfaktoren für eine Abschreibungsentscheidung,

c) der Rechtzeitigkeit und Höhe der Abschreibung,

d) der Wertrelevanz der Abschreibung am Aktienmarkt,

e) dem Ankündigungseffekt für den Aktienmarkt.

ad a): Umfang einer Goodwillaktivierung Über den absoluten Umfang der Goodwillaktivierung informieren insbesondere die jährlichen Untersuchungen von Duff \& Phelps für USA und Europa. Die Europa-Studien von 2017 und 2018 zeigen z. B. für deutsche Gesellschaften im STOXX Europe 600 für die Jahre 2012: 6,0 Mrd. (bei 18 Gesellschaften), 2013: 2,9 Mrd. (17), 2014: 1,4 Mrd. (16), 2015: 11,6 Mrd. (13), 2016: 5,4 Mrd. (8) und 2017: 3,2 Mrd. (13) als Absolutbeträge in Euro. Im Jahr 2016 weisen $98 \%$ dieser Gesellschaften Goodwill aus, aber nur $13 \%$ ein Goodwill Impairment; in 2017 sind es 100\% bzw. $20 \%$ (Duff und Phelps 2018 und 2017, jeweils S. 7; so auch Ballwieser 2019, S. 192).

Der relative Umfang der Aktivierung von Goodwill wird meist durch dessen Bezug auf Bilanzsumme oder Eigenkapital gemessen (Betancourt und Irving 2019; Dreesen 2013; Küting 2013, S. 1796, 2012, S. 1934f.; Rogler et al. 2012, S. 346-349). Hier zeigen sich starke Branchenunterschiede. Ein hoher GoodwillAnteil, der das Eigenkapital stark überschreiten kann, ist typisch für Medien- und Industrieunternehmen, ein geringer für Banken und Versicherungen.

Die akademische Literatur offenbart weiterhin Anhaltspunkte, dass das Management Anreize hat, die planmäßige Abschreibung von Vermögenswerten mit endlicher Nutzungsdauer zu vermeiden. Nach ihr werden durchschnittlich 45-65\% der Anschaffungskosten eines Unternehmens im Rahmen der Kaufpreisaufteilung auf Goodwill zugeteilt (Sellhorn 2019, S. 10). Martin Glaum weist mit Bezug auf mehrere Quellen eine etwas engere Bandbreite von 50 bis $60 \%$ aus (Glaum 2017, S. 27). Das passt z. B. zu den durchschnittlich $59 \%$ bei Shalev et al. in einer Stichprobe mit 320 Beobachtungen aus den Jahren 2001 bis 2008 in den USA (Shalev et al. 2013, S. 827). Dem stehen nur rd. 16\% in der Studie von Glaum et al. mit 9468 unternehmensjahrbezogenen Beobachtungen aus 21 Ländern gegenüber (Glaum et al. 2018, S. 150). Thomas Günther und Christian Ott gelangen in einer Studie mit 51 Großunternehmen aus USA und Europa hingegen zu einem Goodwill-Anteil an Aktiva von 33\% (Günther und Ott 2008, S. 921). Die Zuteilung des Kaufpreises auf Goodwill nimmt mit der relativen Bedeutung eines Bonus im Paket der CEO- 
Entlohnung zu (Shalev et al. 2013, S. 849; Detzen und Zülch 2012) und ist höher, wenn der Aktienkurs mit der Transaktionsankündigung sinkt (Yehuda et al. 2019).

Zurückhaltender gegenüber der Bedeutung der Managementanreize ist Christian Ott. Er geht von der These aus, dass Erstansatz und Wertminderungstest nicht separat untersucht werden dürfen, sondern Entscheidungen über beide Vorgänge verbunden sind, aber noch nicht untersucht wurden. Aus seiner Zufallsstichprobe von 149 Unternehmen, die zwischen 2005 bis 2008 IFRS oder US-GAAP anwendeten, leitet er ab, dass zwar mit einem Wertminderungstest verbundene Freiräume bereits bei Erstansatz von Goodwill berücksichtigt werden. Jedoch liefert seine Untersuchung zugleich Anhaltspunkte, dass der Goodwillansatz nicht nur mit Managementanreizen zusammenhängt, sondern auch Kombinationseffekte aller Vermögenswerte bei Unternehmensfortführung des Zielunternehmens und erwartete Synergien (den ,core goodwill“" nach Johnson und Patrone 1998, S. 294-296; Sellhorn 2010, S. 889) widerspiegelt (Ott 2012, S. 60, 63 und 98). Die beachtliche Höhe der Goodwillaktivierung und deren oftmaliges Übersteigen des buchmäßigen Eigenkapitals bleiben davon unberührt.

ad b): Einflussfaktoren einer Goodwillabschreibung Verschiedene frühe Studien legen bereits nahe, dass Abschreibungen von Goodwill eher auf Anreize des Managements als auf ökonomische Faktoren zurückgehen (Sellhorn 2004; Beatty und Weber 2006). Gegenüber diesen wurden jedoch die Untersuchungen im Hinblick auf Datenbasis und Methodik stark verbreitert. So untersuchen Glaum et al. in einer sehr umfangreichen Studie mit einer multivariaten logistischen Regression die Einflussfaktoren für eine Wertminderungsentscheidung bei IFRS verwendenden börsennotierten Unternehmen aus 21 Ländern. Nach ihnen sind Wertminderungen negativ assoziiert mit ökonomischer Leistung, approximiert durch Aktienrendite, stehen aber auch in Verbindung mit Proxies für management- und unternehmensbezogene Anreize, insbesondere CEO-Wechsel und hohes operatives Ergebnis vor Goodwill-Wertminderung. Die relative Bedeutung von ökonomischer Leistung und Anreizen hängt von der Stärke des nationalen Rechnungslegungs- und Prüfungsvollzugs und von unternehmensbezogenen Eigentümerstrukturen ab. CEO-EntlohnungsBedenken beeinflussen die Entscheidung zur Wertminderung in schwach regulierter Umwelt. CEO-Reputations-Bedenken und eine Präferenz für Gewinnglättung beeinflussen die Entscheidung unabhängig von der Stärke einer Regulierungsdurchsetzung und institutionelle Investoren können die öffentlich-rechtliche Durchsetzung ersetzen (Glaum et al. 2018, S. 149-151).

Die letztgenannten Ergebnisse passen zu jenen von André et al., die in einer Untersuchung der Jahre 2006 bis 2015 feststellen, dass bei im Median ähnlicher Höhe von Goodwill US-Unternehmen gegenüber europäischen Unternehmen zumindest in den Jahren 2008 und 2009 früher Wertminderungen vornehmen und diese einen wesentlich höheren Prozentsatz vom Anfangs-Goodwill eines Jahres ausmachen. „Even though European firms are more likely to impair over multiple years, the cumulative impairments never come close to the level of US firms, be it in a single year or cumulative over multiple years." (André et al. 2016, S. 329.) Man kann davon ausgehen, dass die Rechnungslegungsdurchsetzung in den USA stärker als in Europa ist. 
Ein nicht unwesentlicher Einflussfaktor für Abschreibungsentscheidungen ist die Art der Goodwillzuteilung auf zahlungsmittelgenerierende Einheiten. Die Ergebnisse Binomialer Logistischer Regressionen zeigen, ,dass eine höhere Aggregation von Goodwill in einzelne Berichtssegmente sowie eine Allokation von Goodwill auf größere, profitablere und risikoärmere Berichtssegmente Abschreibungswahrscheinlichkeiten statistisch signifikant reduzieren“ (Eichner 2017, S. 216). Basis waren 154 Beobachtungen von STOXX Europe 600-Unternehmen zwischen 2005 und 2014. Das Ergebnis entspricht den Erwartungen.

Schließlich zeigen Andrei Filip et al. mit Hilfe von Kontrollgruppen, dass bei der Schätzung des beizulegenden Zeitwerts in den USA die Manager die Cash Flow-Prognosen mittels realer Bilanzpolitik nach oben zu treiben scheinen, um eine Wertminderung zu verhindern. Diese Art der Manipulation scheint schädlich für die spätere Unternehmensleistung zu sein (Filip et al. 2015, S. 515).

ad c): Rechtzeitigkeit und Höhe einer Abschreibung Eine auf die Wertminderung bezogene und oft zu hörende Aussage lautet ,too late, too small“. Doch dies dürfte der Wirklichkeit nicht ganz entsprechen, denn nach der bereits erwähnten und breitesten jüngeren Studie von Glaum et al. tendiert die Wertminderung rechtzeitig bei Unternehmen aus Ländern mit hoher Durchsetzungskraft der Regulierung stattzufinden, während Unternehmen in Ländern mit schwacher Regulierungsdurchsetzung in geringerem Ausmaß auf ökonomische Einflussfaktoren einer Wertminderung zu reagieren scheinen (Glaum et al. 2018, S. 149-151).

Davon unberührt stellen Kevin K. Li und Richard G. Sloan fest, dass die Umstellung von SFAS $142 \mathrm{zu}$ relativ weniger zeitgerechten Wertminderungen als früher führte. Es scheint, dass die Beseitigung der planmäßigen Abschreibung, zusammen mit den Schwierigkeiten einer Nachprüfbarkeit des beizulegenden Zeitwerts von Goodwill, zu relativ stark aufgeblähten Goodwillansätzen und relativ weniger rechtzeitigen Wertminderungen geführt hat (Li und Sloan 2017, S. 965).

Von Interesse in diesem Zusammenhang ist schließlich eine von Jeffery Abarbanell betreute Dissertation über die Auslöser von Impairment Tests. Ihre Verfasserin zeigt anhand einer Faktoranalyse, dass diese als unternehmens-, branchen- und wirtschaftsbezogen unterteilt werden können, und findet am Aktienmarkt signifikante Preis- und Volumenreaktionen nach einer Entscheidung zur Wertminderung. Dies gilt aber nur bei unternehmensbezogenen Auslösern. Die fehlende Unterscheidung der Auslöser könnten nach ihrer Auffassung die gemischten Resultate früherer Untersuchungen erklären. „Additional findings indicate (...), firm-specific triggering events increase the post-announcement information asymmetry, and predict future goodwill impairments when a firm records multiple impairments“ (Nykyforovych 2017, Abstract).

ad d): Wertrelevanz am Aktienmarkt Wertrelevanz am Aktienmarkt wird verstanden als Assoziation zwischen Größen der Rechnungslegung und Aktienkursen. Die Höhe der Korrelation zwischen Kapitalmarktrenditen und Gewinnen dient als Beurteilungskriterium für die Rechnungslegungsgüte (Wagenhofer und Ewert 2015, S. 118-120). 
Sascha Boennen und Martin Glaum geben einen Überblick über einige Studien, die prüften, ob die Wertrelevanz von Goodwill sich durch Verwendung von SFAS 142 und IAS 36 (rev. 2004) geändert hat (Boennen und Glaum 2014, S. 20-23). Sie finden hierfür einige Anhaltspunkte mit Daten aus Australien (Chalmers et al. 2008), Portugal (Oliviera et al. 2010) und Großbritannien (Horten und Serafeim 2010), aber keine ganz überzeugende Evidenz (vgl. a. Beyer 2015, S. 254-257).

ad e): Ankündigungseffekt am Aktienmarkt Thorsten Knauer und Arnt Wöhrmann testen mit Hilfe einer Ereignisstudie den Ankündigungseffekt einer Goodwillwertminderung mit einer Stichprobe von 564 Beobachtungen zwischen 2005 und 2009. Sie finden negative Kapitalmarktwirkungen bei unerwarteten Ankündigungen. Ihre Ergebnisse zeigen, dass dieser Effekt umso größer ist, je schwächer der Investorenschutz eines Landes ist und je mehr Spielräume dem Management verbleiben (Knauer und Wöhrmann 2016; zu früheren Studien vgl. Schatt et al. 2016, S. 319).

\subsection{Würdigung der Diskussion}

Die im ersten Perspective Paper unterbreitete Argumentation ist aus mehreren Gründen fragwürdig. Zentral ist dort die Aussage, dass Goodwill ein sich nicht verzehrender Vermögenswert deshalb sein müsse, weil sonst die bei Unternehmensbewertungen üblicherweise angenommenen künftig steigenden Cash Flows nicht zu erklären seien. Zum einen gibt es jedoch mehrere rechentechnische sowie potentiell marktund unternehmensbezogene Gründe, was künftig steigende Cash Flows erklären könnte. Dazu zählen die Nominalrechnung bei Geldentwertung, eine potentiell zunehmend bessere Marktstellung dank Konzentrationsprozessen mit Skaleneffekten dank M\&A, ein eventuell vorliegendes natürliches Monopol, z. B. in der Netzindustrie, eine verbesserte Effektivität und effizientere Nutzung von Vermögenswerten, unabhängig von ihrer Abnutzbarkeit, und selbst erzeugte verstärkte Synergien. Zum anderen kann in den Jahren nach Unternehmenserwerb oder Verschmelzung originärer Goodwill erzeugt worden sein: durch Ansiedlungspolitik mit Standortvorteilen, verbesserte Gewinnung von Talenten, Mitarbeiterschulung, Forschungs- und Entwicklungsaktivitäten u.a.m.

Ferner hat Bilanzierung mit Unternehmensbewertung keine direkte theoretische Verbindung. Z.B. sind ertrags- oder unternehmenswertabhängige Abschreibungen verboten. Bilanzierung kann auch keine Unternehmensbewertung auf Basis einer Cash Flow-Prognose ersetzen und nur sehr begrenzt zur Cash Flow-Prognose beitragen. Diesbezügliche Ansätze hat die theoretische Literatur zwar insbesondere zu Beginn des 20. Jahrhunderts entwickelt, aber erkennbar ohne Erfolg (Moxter 1984 und 1982). Wer dies noch immer bezweifelt, mag nur einen Blick auf die Diskrepanz von buchmäßigem Eigenkapital und Marktkapitalisierung bei börsennotierten Unternehmen werfen (nicht nur bei prominenten deutschen Banken), wobei nicht gesagt wird, dass die Marktkapitalisierung dem Unternehmenswert oder einem potentiellen Kaufpreis entspricht.

Die im zweiten Perspective Paper unterbreiteten Zahlenbeispiele sind unstrittig fehlerfrei, bauen aber durchgängig auf einer ausgesprochen kritischen Annahme auf. 
Danach kennt man nach Unternehmenserwerb über mehrere (hier: fünf) Jahre den Fair Value und den nach Erwerb nicht immer konstant bleibenden Buchwert des erworbenen Unternehmens und der auf Goodwill zu testenden Einheit. Die in praxi üblichen, in möglichst wenigen Jahren erfolgenden Integrationsversuche und -erfolge von erworbenen oder verschmolzenen Unternehmen werden dadurch negiert. Es ist klar, dass jede modellhafte Überlegung von der Realität abweichen muss. Die hier gesetzte Annahme droht jedoch aufgrund eines unrealistischen Idealfalls die Untauglichkeit der Zahlenbeispiele für fundierte politische Entscheidungen nahezulegen, zumal sich durch andere Zahlenannahmen genauso gut ein Informationswert des IOA hätte zeigen lassen.

Freilich ist Letzteres auch gar nicht das Ziel in diesem Paper. Das Paper zeigt mit anderen Worten keineswegs einen vorlaufenden Informationswert, sondern das Gegenteil: vier Gründe für dessen Beeinträchtigung. Um den Informationswert im Sinne eines ,leading indicator“ zu belegen, hätte es doch der intensiveren Diskussion der widersprüchlichen Ergebnisse der akademischen Studien bedurft. Mit der Aufnahme von kurzen Ergebnisbeschreibungen der Resultate von Li et al. (2011), Knauer und Wöhrmann (2016), Li und Sloan (2017) sowie Nykyforovych (2017) in den Anhang kann der Leser das Urteil nachzuziehen versuchen, wonach sich die Behauptung, dass , in certain instances goodwill impairments are undoubtably a leading indicator" (IVSC 2019h, S. 4), wirklich belegen lässt. Li et al. (2011) mögen hierbei helfen, während die sonst genannten Studien keineswegs eindeutige Resultate liefern, sondern diese immer an bestimmte Nebenbedingungen geknüpft sind. Mit dem Bezug auf „,certain instances“ wird das zwar möglicherweise angedeutet, dies reicht aber nicht dafür, das Ziel des Informationswerts insgesamt zu belegen.

Diese Einwendungen führen zu der Schlussfolgerung, dass auch die dritte Frage unseres Beitrags negativ zu beantworten ist: Die in den beiden Perspective Papers des IVSC enthaltenen Argumentationslinien über die Abnutzbarkeit von aktiviertem Goodwill und den Informationswert von Wertminderungen haben keine ökonomisch überzeugende Begründung. Das erste Paper verbindet auf nicht nachvollziehbare Weise Bilanzierung mit Unternehmensbewertung. Das zweite Paper erkennt die widersprüchlichen empirischen Ergebnisse akademischer Arbeiten und wählt Beispielrechnungen, um den Nachteil des IOA in bestimmten Situationen zu dokumentieren. Die Rechnungen sind unstrittig, aber die Annahme eines bekannten zukünftigen Fair Value von erworbenen Unternehmen geht an der Praxis so stark vorbei, dass der Wert dieser Beispiele immens beeinträchtigt wird. Auch wird nicht etwa der Informationswert einer Wertminderung im Sinne eines vorlaufenden Signals, sondern beispielhaft genau das Gegenteil belegt. Die Konsequenzen für eine politische Entscheidung pro oder contra IOA sind damit unbestimmt.

Unbestritten ist, dass ein Wechsel von IOA zu planmäßiger Abschreibung von Goodwill ungemein hohe wertmäßige Konsequenzen für Bilanz und GuV hätte, was insbesondere das CFA Institute in den USA auf die Invitation to Comment des FASB zu Recht herausgestellt hat. Wenn man allein aus diesem Grund auf eine Umstellung auf die planmäßige Abschreibung von Goodwill verzichtet, muss man sehen, dass dies nicht ökonomisch begründbar ist. Vielmehr würde der seinerzeit aus dem Verbot der Interessenzusammenführung folgende IOA dann dazu führen, ein fragwürdiges Verfahren allein aus Umstellungsproblemen fortzuführen. Damit wird nicht nur die 
Entscheidungsnützlichkeit von Konzernabschlüssen fraglich, sondern auch Erstellern, Prüfern und Compliance-Institutionen viel zugemutet. Die Zumutung dürfte kaum geringer werden, wenn der IOA durch erhöhte Informationspflichten verbessert werden sollte. Die Frage, wann Unternehmenskäufe oder Verschmelzungen erfolgreich sind, ist sowohl mit bilanziellen Daten als auch mit Kapitalmarktdaten nach Ankündigung oder Realisierung der Transaktion bekanntermaßen nur schwer zu beantworten. Aber genau darum müsste es bei den Verbesserungsvorschlägen gehen.

Gleichermaßen unbestritten fällt eine begründete Nutzungsdauerschätzung von Goodwill schwer. Deshalb hat man sich rechtlich stets mit Typisierungen beholfen: mit Vorgaben von bis zu 40, 20 oder 10 Jahren (so über verschiedene Zeiträume in den USA), mit 10 Jahren, wenn die Nutzungsdauer nicht verlässlich geschätzt werden kann ( $\$ 253$ Abs. 3 Satz 3 HGB), oder mit 15 Jahren ( 7 Abs. 1 Satz 3 EStG). Das gilt schließlich auch für andere Vermögenswerte wie Gebäude oder Elemente der Betriebs- und Geschäftsausstattung.

Die Vorgabe solcher Typisierungen auch für Goodwill hätte aus unserer Sicht einen bekannten, aber nichtsdestoweniger entscheidenden Vorteil: Sie würde den darin bestehenden Widerspruch beseitigen, dass nach allen uns bekannten Rechtsregeln (US-GAAP, IFRS, HGB etc.) die Aktivierung von originärem Goodwill aus Gründen zweifelhafter Werthaltigkeit verboten ist, während sie durch den IOA in Jahren nach Unternehmenserwerb oder Verschmelzung erlaubt wird.

\section{Zusammenfassung}

Mit dem IVSC ist eine seit Mitte des letzten Jahrzehnts immer aktiver werdende nichtstaatliche Organisation entstanden, die weltweit einheitliche Bewertungsregeln für Vermögenswerte und Unternehmen etablieren möchte. Zielsetzung ist Harmonisierung und Vergleichbarkeit. Sie greift ferner aktuell in die Diskussion der bilanziellen Goodwill-Folgebewertung nach US-GAAP und IFRS ein und versucht, die politische Diskussion über dieses Thema mit zu beeinflussen.

Im vorliegenden Beitrag haben wir erstens geprüft, ob das IVSC eine dem IASB oder der IOSCO vergleichbare Entwicklung nehmen und Bedeutung erhalten könnte. Dies ist aus mehreren Gründen zu verneinen. Neben Manpower und Finanzierung fehlt der Versuch der Ausrichtung der Bewertungsstandards an einem Ziel, wie es den IFRS vergleichbar ist. Das IVSC sammelt bisher relativ allgemeingehaltene Bewertungsgrundlagen und -verfahren, ohne sie stringent zu ordnen und auf Ableitbarkeit, Widerspruchsfreiheit oder Komplementarität zu prüfen.

Zweitens fragten wir, welche Schlussfolgerungen sich hinsichtlich der potentiellen Verbindlichkeit von IVS oder ihres freiwilligen Orientierungsnutzens für Deutschland ziehen lassen, d.h. welcher Bedarf an IVS besteht. Auch dies wurde mit mehreren Gründen verneint. Es fehlt an einer aussagekräftigen Systematisierung von Bewertungszwecken, was eine zweckgerechte Ableitung von Handlungsempfehlungen für die Bewertung, wie sie insbesondere in Deutschland vorhanden und erkennbar ist, behindert. Die Bewertungsgrundlagen und -verfahren werden in Form von Hinweisen auf Existenz und Varianten geschildert, ohne einem Bewerter Hand- 
lungsempfehlungen für bestimmte Situationen zukommen zu lassen. Der potentielle Wert einer Bestätigung, die IVS eingehalten zu haben, wird dadurch beeinträchtigt und kann allenfalls im Rahmen der Argumentationsfunktion wertvoll sein.

Da sich das IVSC auch zur aktuellen Diskussion der Folgebewertung von Goodwill äußert, haben wir drittens gefragt, ob und inwieweit die Argumentationen ökonomisch fundiert sind. Auch das führte zu keinem positiven Ergebnis. Die Begründung für einen nicht abnutzbaren Goodwill war ebenso wenig überzeugend wie die beispielhaften Versuche, dem IOA einen Informationswert als vorlaufender Indikator in einigen Fällen zuzusprechen. Das IVSC begründet die fehlende Abnutzbarkeit von aktiviertem Goodwill damit, dass anders die für Zwecke der Unternehmensbewertung oftmals angenommenen wachsenden Cash Flows nicht zu begründen seien. Diese Argumentation ist einseitig und vernachlässigt rechentechnische, markt- und unternehmensbezogene Aspekte. Der nach US-GAAP und IFRS geltende IOA leidet ferner unter dem Widerspruch, dass national die Aktivierung von originärem Goodwill verboten ist, während sie durch den IOA nach Unternehmenserwerb oder Verschmelzung erlaubt wird. Zugleich zeigt die akademische Literatur, dass es viele Anhaltspunkte gibt, wonach das Management beim IOA seine Freiheitsgrade ausnutzt und dass die Wertminderung nicht zeitgerecht erfolgt. Sein Informationswert erscheint insofern begrenzt. Die vom IVSC in einem Perspective Paper verwendeten Beispiele gehen immer von der Annahme aus, dass in bestimmten Fällen die Wertminderungen unzweifelhaft ein für den Kapitalmarkt vorauseilender Indikator sind, und widmen sich der Frage, wann diese Eigenschaft fehlen könnte. Sie sind deshalb auf dem Weg, Verbesserungen des IOA zu suchen, ohne auf die stark gemischten Ergebnisse der ökonomischen Untersuchungen zur Indikatoreigenschaft von Wertminderungen vertieft einzugehen. Damit bleibt offen, wie die Argumentation zur politischen Entscheidung über Abschaffung oder Verbesserung des IOA wirklich unterstützt werden kann.

Funding Open Access funding provided by Projekt DEAL.

Open Access Dieser Artikel wird unter der Creative Commons Namensnennung 4.0 International Lizenz veröffentlicht, welche die Nutzung, Vervielfältigung, Bearbeitung, Verbreitung und Wiedergabe in jeglichem Medium und Format erlaubt, sofern Sie den/die ursprünglichen Autor(en) und die Quelle ordnungsgemäß nennen, einen Link zur Creative Commons Lizenz beifügen und angeben, ob Änderungen vorgenommen wurden.

Die in diesem Artikel enthaltenen Bilder und sonstiges Drittmaterial unterliegen ebenfalls der genannten Creative Commons Lizenz, sofern sich aus der Abbildungslegende nichts anderes ergibt. Sofern das betreffende Material nicht unter der genannten Creative Commons Lizenz steht und die betreffende Handlung nicht nach gesetzlichen Vorschriften erlaubt ist, ist für die oben aufgeführten Weiterverwendungen des Materials die Einwilligung des jeweiligen Rechteinhabers einzuholen.

Weitere Details zur Lizenz entnehmen Sie bitte der Lizenzinformation auf http://creativecommons.org/ licenses/by/4.0/deed.de. 


\section{Literatur}

André, Paul, Andrei Filip, und Luc Paugam. 2016. Examining the patterns of goodwill impairments in europe and the US. Accounting in Europe 13:329-352.

Ballwieser, Wolfgang. 2019. Überlegungen zu Theorie und Praxis der Finanzberichterstattung: Herausforderungen, Erkenntnisse und Lücken. Zeitschrift für betriebswirtschaftliche Forschung 71:169-211.

Ballwieser, Wolfgang, und Dirk Hachmeister. 2016. Unternehmensbewertung - Prozess, Methoden und Probleme, 5. Aufl., Stuttgart: Schäffer-Poeschel.

Beatty, Anne, und Joseph Weber. 2006. Accounting discretion in fair value estimates: an examination of SFAS 142 goodwill impairments. Journal of Accounting Research 44:257-288.

Betancourt, Luis, und James H. Irving. 2019. The challenge of accounting for goodwill. Impact of a possible return to amortization. The CPA Journal. https://www.cpajournal.com/2019/11/27/the-challengeof-accounting-for-goodwill/. Zugegriffen: 17. Dez. 2019.

Beyer, Bettina. 2015. Die Bilanzierung des Goodwills nach IFRS. Eine konzeptionelle Betrachtung von Ansatz, Erst- und Folgebewertung. Wiesbaden: Springer Gabler.

Boennen, Sascha, und Martin Glaum. 2014. Goodwill accounting: a review of the literature. https://papers. ssrn.com/sol3/papers.cfm?abstract_id=2462516. Zugegriffen: 17. Dez. 2019.

Chalmers, Keryn, Greg Clinch, und Jayne M. Godfrey. 2008. Adoption of International Financial Reporting Standards: Impact on the Value Relevance of Intangible Assets. Australian Accounting Review 18:237-247.

Coenenberg, Adolf, und Günter Sieben. 1976. Unternehmungsbewertung. In Handwörterbuch der Betriebswirtschaft, 4. Aufl., Hrsg. Erwin Grochla, Waldemar Wittmann, 4062-4079. Stuttgart: SchäfferPoeschel.

Detzen, Dominic, und Henning Zülch. 2012. Executive Compensation and Goodwill Recognition under IFRS: Evidence from European Mergers. Journal of International Accounting, Auditing and Taxation 21:106-126.

Dreesen, Heinz. 2013. Beurteilung des Goodwill-Accounting nach IFRS unter quantitativen und qualitativen Aspekten. Eine empirische Studie am Beispiel der SDAX-Unternehmen. Zeitschrift für internationale und kapitalmarktorientierte Rechnungslegung 13:469-478.

Duff \& Phelps. 2017. 2017 European Goodwill Impairment Study. https://www.slideshare.net/Duffand Phelps/2017-european-goodwill-impairment-study?from_action=save. Zugegriffen: 10. Febr. 2019.

Duff \& Phelps. 2018. 2018 European Goodwill Impairment Study. https://www.duffandphelps.co.uk/ insights/publications/goodwill-impairment/2018-european-goodwill-impairment-study. Zugegriffen: 6. Apr. 2019.

Eichner, Korbinian. 2017. Goodwill-Konzentration und -Abschreibungswahrscheinlichkeiten. Ein problematischer Zusammenhang bei der Werthaltigkeitsprüfung von Goodwill. Internationale und kapitalmarktorientierte Rechnungslegung 17:208-216.

FASB. 2014. Intangibles_goodwill and other (topic 350). https://asc.fasb.org/imageRoot/79/49129379. pdf. Zugegriffen: 16. Dez. 2019.

FASB. 2018. Not-for-profit entities. (topic 958). https://asc.fasb.org/imageRoot/62/117396462.pdf. Zugegriffen: 16. Dez. 2019.

FASB. 2019a. Identifiable intangible assets and subsequent accounting for goodwill. https://www.fasb. org/jsp/FASB/Document_C/DocumentPage?cid=1176172950529\&acceptedDisclaimer=true. Zugegriffen: 3. Dez. 2019.

FASB. 2019b. Online comment letters. https://www.fasb.org/jsp/FASB/CommentLetter_C/CommentLetter Page\&cid=1218220137090\&project_id=2019-720. Zugegriffen: 16. Dez. 2019.

Filip, Andrei, Thomas Jeanjean, und Luc Paugam. 2015. Using real activities to avoid goodwill impairment losses: evidence and effect on future performance. Journal of Business Finance and Accounting 42:515-554.

Glaum, Martin, Wayne R. Landsman, und Sven Wyrwa. 2018. Goodwill impairment: the effects of public enforcement and monitoring by institutional investors. The Accounting Review 93:149-180.

Glaum, Martin. 2017. Goodwill impairment - an academic perspective. 16. IFRS Kongress, Berlin, 7. September 2017. https://www.ey.com/Publication/vwLUAssets/ey-002-glaum-goodwill-impairmentan-academic-perspective/\$FILE/ey-002-glaum-goodwill-impairment-an-academic-perspective.pdf. Zugegriffen: 3. Dez. 2019.

Gonnella, Enrico, und Lucia Talarico. 2018. Regulation in business valuation: the case of the international valuation standards council. Research Journal of Finance and Accounting 9:102-115. 
Günther, Thomas, und Christian Ott. 2008. Behandlung immaterieller Ressourcen bei Purchase Price Allocations: Ergebnisse einer explorativen empirischen Studie. Die Wirtschaftsprüfung 61:917-926.

Hayn, Carla, und Patrica J. Hughes. 2006. Leading indicators of goodwill impairment. Journal of Accounting, Auditing and Finance 21:223-265.

Horton, Joanne, und George Serafeim. 2010. Market reaction to and valuation of IFRS reconciliation adjustments: first evidence from the UK. Review of Accounting Studies 15:725-751.

IASB. 2015. AP18B. Feedback from users of financial statements about information on goodwill and impairment. November 2015. https://cdn.ifrs.org/-/media/feature/meetings/2015/november/iasb/ goodwill-and-impairment/ap18b-goodwill-and-impairment-project.pdf. Zugegriffen: 3. Dez. 2019.

IASB. 2016. AP18. Cover Paper, includes feedback from the 2015 Agenda Consultation. May 2016. https:// cdn.ifrs.org/-/media/feature/meetings/2016/may/iasb/goodwill-and-impairment/ap18-goodwill-andimpairment.pdf. Zugegriffen: 3. Dez. 2019.

IDW. 2008. IDW Standard: Grundsätze zur Durchführung von Unternehmensbewertungen (IDW S 1 i.d.F. 2008) (Stand: 02.04.2008). Die Wirtschaftsprüfung 61(Supplement 3):68-89.

IFRS. 2019. Who uses IFRS standards? https://www.ifrs.org/use-around-the-world/use-of-ifrs-standardsby-jurisdiction/. Zugegriffen: 27. Nov. 2019.

IOSCO. 2019. Fact-Sheet July 2019. https://www.iosco.org/about/pdf/IOSCO-Fact-Sheet.pdf. Zugegriffen: 27. Nov. 2019.

IVS. 2020. International Valuation Standards (IVS). Effective 31 January 2020. London; per Subskription beziehbar über https://www.ivsonline.org/.

IVSC. 2014. Statement of protocols for co-operation on international financial reporting standards and international valuation standards between the international valuation standards council and the IFRS foundation, 6 march 2014. https://www.ifrs.org/-/media/feature/around-the-world/memoranda/ivscprotocol.pdf. Zugegriffen: 27. Nov. 2019.

IVSC. 2017. International valuation standards 2017. https://www.ivsc.org/standards/international-valuationstandards. Zugegriffen: 27. Nov. 2019.

IVSC. 2018. IVS 2017 proposed revisions exposure draft. https://www.ivsc.org/files/file/view/id/1444. Zugegriffen: 27. Nov. 2019.

IVSC. 2019a. IVS 220 non-financial liabilities exposure draft. https://www.ivsc.org/files/file/view/id/1345. Zugegriffen: 27. Nov. 2019.

IVSC. 2019b. IVS 2017 additional technical revisions exposure draft. https://www.ivsc.org/files/file/view/ id/1409. Zugegriffen: 27. Nov. 2019.

IVSC. 2019c. Consultation responses. https://www.ivsc.org/standards/international-valuation-standards/ consultation/proposed-revisions-to-ivs-2017\#tab-responses. Zugegriffen: 27. Nov. 2019.

IVSC. 2019d. Consultation responses. https://www.ivsc.org/standards/international-valuation-standards/ consultation/ivs-2017-additional-technical-revisions-exposure-draft\#tab-responses. Zugegriffen: 27. Nov. 2019.

IVSC. 2019e. IVS: basis for conclusions-reflecting changes introduced to IVS (effective 31 january 2020). https://www.ivsc.org/files/file/view/id/1608. Zugegriffen: 27. Nov. 2019.

IVSC. 2019f. Annual report 2018-19. https://www.ivsc.org/about/annual-reports. Zugegriffen: 27. Nov. 2019.

IVSC. 2019g. Is goodwill a wasting asset? https://www.ivsc.org/news/article/is-goodwill-a-wasting-asset. Zugegriffen: 27. Nov. 2019.

IVSC. 2019h. Information value of the current impairment test: leading or lagging indicator? https://www. ivsc.org/files/file/view/id/1675. Zugegriffen: 11. Febr. 2020.

Johnson, L. Todd, und Kimberley R. Patrone. 1998. Is Goodwill an Asset? Accounting Horizons 12:293-303.

Kleekämper, Heinz. 2000. IASC - Das Trojanische Pferd der SEC? In US-amerikanische Rechnungslegung - Grundlagen und Vergleiche mit deutschem Recht, Bd. 4, Hrsg. Wolfgang Ballwieser, 467-484. Stuttgart: Schäffer-Poeschel.

Knauer, Thorsten, und Arnt Wöhrmann. 2016. Market reaction to goodwill impairments. European Accounting Review 25:421-449.

Küting, Karlheinz. 2012. Der Geschäfts- oder Firmenwert in der deutschen Konsolidierungspraxis 2011. Ein Beitrag zur empirischen Rechnungslegungsforschung. Deutsches Steuerrecht 50:1932-1939.

Küting, Karlheinz. 2013. Der Geschäfts- oder Firmenwert in der deutschen Konsolidierungspraxis 2012. Ein Beitrag zur empirischen Rechnungslegungsforschung. Deutsches Steuerrecht 51:1794-1803.

Li, Kevin K., und Richard G. Sloan. 2017. Has goodwill accounting gone bad? Review of Accounting Studies 22:964-1003. 
Li, Zining, Pervin K. Shroff, Ramgopal Venkataraman, und Ivy Xiying Zhang. 2011. Causes and consequences of goodwill impairment losses. Review of Accounting Studies 16:745-778.

Moxter, Adolf. 1982. Betriebswirtschaftliche Gewinnermittlung. Tübingen: J.C.B. Mohr.

Moxter, Adolf. 1983. Grundsätze ordnungsmäßiger Unternehmensbewertung, 2. Aufl., Wiesbaden: Gabler.

Moxter, Adolf. 1984. Einführung in die Bilanztheorie, 3. Aufl., Bilanzlehre, Bd. I. Wiesbaden: Gabler.

Nykyforovych, Maria. 2017. Trigger Warnings: When is Goodwill Impairment Disclosure Informative? Ph.D. Diss., Chapel Hill 2017. https://pdfs.semanticscholar.org/e0cd/06224109bebae4471cac895e908 72229707a.pdf. Zugegriffen: 17. Dez. 2019.

Oliviera, Lidia, Lucia Lima Rodrigues, und Russell Craig. 2010. Intangible assets and value relevance: evidence from the Portuguese stock exchange. The British Accounting Review 42:241-252.

Ott, Christian. 2012. Mergers \& Acquisitions and Intangibles. Dissertation Technische Universität Dresden. https://pdfs.semanticscholar.org/e2b0/3d5f5d17257520db878df497b78644c1d6a3.pdf. Zugegriffen: 17. Dez. 2019.

Ramanna, Karthik. 2008. The implications of unverifiable fair-value accounting: Evidence from the political economy of goodwill accounting. Journal of Accounting and Economics 45:253-281.

Rogler, Silvia, Sandro Veit Straub, und Martin Tettenborn. 2012. Bedeutung des Goodwill in der Bilanzierungspraxis deutscher kapitalmarktorientierter Unternehmen. Zeitschrift für internationale und kapitalmarktorientierte Rechnungslegung 12:343-351.

Schatt, Alain, Leonidas Doukakis, Corinne Bessieux-Ollier, und Elisabeth Walliser. 2016. Do goodwill impairments by European firms provide useful information to investors? Accounting in Europe 13:307-327.

Sellhorn, Thorsten. 2004. Goodwill-Impairment-An Empirical Investigation of Write-Offs under SFAS 142. Frankfurt am Main: Peter Lang.

Sellhorn, Thorsten. 2010. Ansätze zur bilanziellen Behandlung des Goodwill im Rahmen einer kapitalmarktorientierten Rechnungslegung. Der Betrieb 53:885-892.

Sellhorn, Thorsten. 2019. Goodwill impairment test. 305. Sitzung in München, 11. Nov. 2019. Arbeitskreis Externe Unternehmensrechnung der Schmalenbach-Gesellschaft für Betriebswirtschaft e. V..

Shalev, Ron, Ivy Xiying Zhang, und Yong Zhang. 2013. CEO compensation and fair value accounting: evidence from purchase price allocation. Journal of Accounting Research 51:819-854.

Sieben, Günter. 1966. Der Anspruch auf angemessene Abfindung nach § 12 UmwG. Höchstrichterliche Entscheidungen in betriebswirtschaftlicher Sicht. Die Aktiengesellschaft 11:6-13, 54-78 und 83-89.

Sieben, Günter. 1977. Eröffnung der Tagung. In Moderne Unternehmungsbewertung und Grundsätze ihrer ordnungsmäßigen Durchführung, Hrsg. Wolfgang Goetzke, Günter Sieben, 27-31. Köln: GeberaSchriften.

Talbot, Nick. 2019. IVSC and the evolution of the BV profession. OIV presentation. https://www. fondazioneoiv.it/wp-content/uploads/2019/11/Talbot_OIV-Presentation_compressed_compressed1-2.pdf. Zugegriffen: 27. Nov. 2019.

Tönnes, Wolf-Achim. 2019. Internationalisierung der Unternehmensbewertung. In Unternehmensbewertung. Festschrift für Bernhard Großfeld zum 85. Geburtstag, Hrsg. Wolf-A. Tönnes, 429-450. Heidelberg: C.F. Müller.

Wagenhofer, Alfred, und Ralf Ewert. 2015. Externe Unternehmensrechnung, 3. Aufl., Berlin, Heidelberg: Springer Gabler.

Yehuda, Nir, Linda Vincent, und Thomas Lys. 2019. The nature and implications of acquisition goodwill. Asia-Pacific Journal of Accounting \& Economics 26:709-730. 